LS-53 (ANL)
$8 / 5 / 85$

Y. Cho

\title{
Optimization of $B$ Functions through Insertion Devices
}

Introduction

It is generally noted that at an undulator straight section, the horizontal beta function is made to be large while the vertical beta function is relatively small. On the other hand, at a wiggler straight section, both horizontal and vertical beta functions are made to be small. In this note we describe a procedure with which optimum settings of the beta functions in the insertion straight section are to be determined. For this we consider separately for the undulator radiation and the radiation from the wiggler device.

Since the brilliance of radiation is a canonically conserved quantity, we use the brilliance as a figure of merit for the consideration. Then the optimization process is to find a set of horizontal and vertical beta functions which would give the maximum brilliance when the natural emittance, emittance coupling constant, length of insertion device and the photon energy to which the insertion device is optimized are specified. The next step of the study is to find sensitivities of the beta functions to the brilliance.

After having done these, we will attempt to find a set of universally optimized beta functions with which all of given kind of the insertion device, e.g., all undulators or all wigglers can be operated. The purpose of this attempt is that if all undulators, regardless of photon energies for which these are optimized, can have the same beta functions, the machine lattice becomes quite simpler and can maintain higher periodicity.

In order to make the description of this study complete, we review some of definitions used herein.

Coupling Constant k: Coupling constant is defined to be

$$
\begin{aligned}
& \varepsilon_{x}=\varepsilon_{x o} / \sqrt{1+k} \\
& \varepsilon_{y}=k \varepsilon_{x o} / \sqrt{1+k}
\end{aligned}
$$




\section{DISCLAIMER}

Portions of this document may be illegible in electronic image products. Images are produced from the best available original document. 


\section{DISCLAIMER}

This report was prepared as an account of work sponsored by an agency of the United States Government. Neither the United States Government nor any agency thereof, nor any of their employees, makes any warranty, express or implied, or assumes any legal liability or responsibility for the accuracy, completeness, or usefulness of any information, apparatus, product, or process disclosed, or represents that its use would not infringe privately owned rights. Reference herein to any specific commercial product, process, or service by trade name, trademark, manufacturer, or otherwise does not necessarily constitute or imply its endorsement, recommendation, or favoring by the United States Government or any agency thereof. The views and opinions of authors expressed herein do not necessarily state or reflect those of the United States Government or any agency thereof. 
where $\varepsilon_{x o}$ is the natural emittance of the storage ring, and $\varepsilon_{x}$ and $\varepsilon_{y}$ are the resultant horizontal and vertical emittances due to the coupling of $k$, respectively.

Photon flux is defined as number of photons/sec/eV of photon or number of photons $/ \mathrm{sec} / 0.1 \% \mathrm{BW}$.

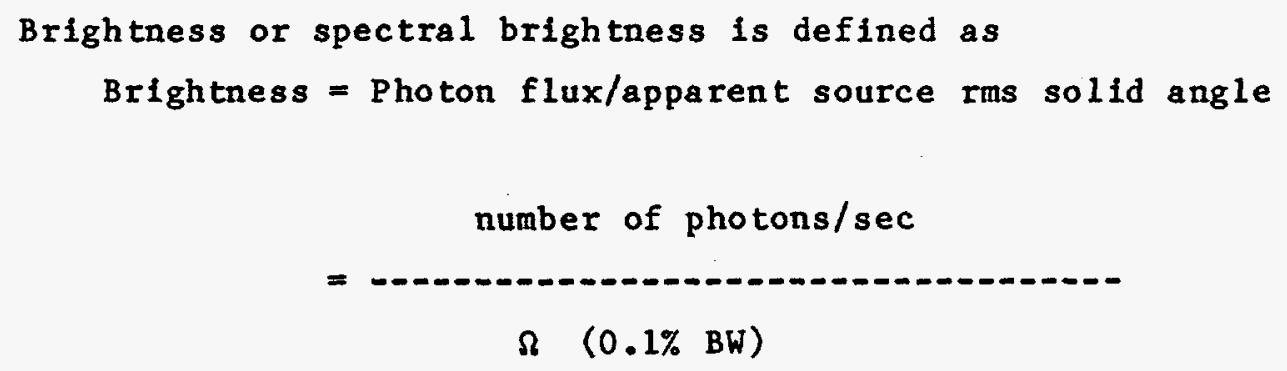

where $\Omega$ is the apparent rms solid angle of the source:

and

$$
\Omega=2 \pi S_{x}^{\prime} S_{y}^{\prime} \quad\left(\operatorname{mrad}^{*} * 2\right)
$$$$
s_{x, y}^{\prime}=\sqrt{\sigma_{x y}^{\prime 2}+\lambda / L}
$$

Here, $S_{x}^{\prime}$ and $S_{y}^{\prime}$ are the apparent divergences of the radiation from the insertion device in the $x$ and $y$ directions, and $\sigma_{x}^{\prime}$ and $\sigma_{y}^{\prime}$ are the rms divergences of the electron beam in the $x$ and $y$ planes through the insertion device, and $\lambda$ is the wavelength of the photon beam.

$$
\begin{aligned}
& \sigma_{x}^{\prime}=\sqrt{\varepsilon_{x} / \beta_{x}} \\
& \sigma_{y}^{\prime}=\sqrt{\varepsilon_{y} / \beta_{y}}
\end{aligned}
$$

The brilliance is then defined to be:

$$
\text { Brilliance }=\quad \text { number of photons/sec }
$$

where $S$ is the rms source area, 


$$
\begin{aligned}
& S=2 \pi S_{x} S_{y} \\
& S_{x}=\sqrt{\sigma_{x}^{2}+\left(\lambda L+\sigma_{x}^{\prime 2} L^{2}\right) / 4} \\
& S_{y}=\sqrt{\sigma_{y}^{2}+\left(\lambda L+\sigma_{y}^{\prime 2} L^{2}\right) / 4} \\
& \sigma_{x}=\sqrt{\beta \varepsilon_{x}} \\
& \sigma_{y}=\sqrt{\beta \varepsilon_{y}}
\end{aligned}
$$

Uundulator Optimization:

Under the given parameters of $\varepsilon_{x o}, k$, and $\lambda$, the goal is to obtain the minimum value of $S \cdot \Omega$ as the function of beta-x and beta-y. In order to demonstrate the sensitivities $S \cdot \Omega$ which is the four dimensional transverse phase space with respect to the beta functions, we make contour plots of $1 /(S \cdot \Omega)$ as a function of beta-x and beta-y.

Figures 1 - 6 show the inverse of the phase spaces for various photon energies $(1 \sim 20 \mathrm{keV})$. The contours are normalized to the maximum value which is makred with an "H", and each contour line is plot with an interval of $2 \%$ from the previous. In another word, the inner most contour represents 98 percentile and the outer contour represents the 90 percentile contour. Also shown in the figures is the phase space value at the peak in unit of meter squared. The values at null beta functions are calculated at beta $=0.1 \mathrm{~m}$.

Figure 7 shows the result of summing all six contours and renormalized by dividing by 6 . This is an attempt to find a universal setting of the beta functions with which all undulator could be efficient.

\section{Wiggler Consideration}

Detalled consideration of the brightness of wiggler shows that

$$
\text { Brightness }=2 \mathrm{~N} \cdot 3.461 \times 10^{6} \gamma^{2} I\left(\frac{\varepsilon}{\varepsilon_{c}}\right)^{2} k_{2 / 3}{ }^{2}\left(\frac{\varepsilon}{2 \varepsilon_{c}}\right)
$$

which is independent of the wiggler geometry. Therefore, to optimize the 
brilliance of wiggler, we optimize the quantity $S$. We have studied this for the photon energies up to $40 \mathrm{keV}$, and the results are shown in Figure 8 . Notice that the brilliance for the wiggler is independent of the photon energy.

\section{Conclusion}

This simple study shows that for about 5 meter undulator, operating with $\varepsilon_{x o}=\gamma \lambda 10^{-9} \mathrm{~m}$ and $\mathrm{k}=0.1, \beta_{x}=12 \mathrm{~m}$ and $\beta_{y}=6 \mathrm{~m}$ would provide an optimum undulator radiations from $1-20 \mathrm{keV}$ range. For wiggler radiation, the beta function setting should be around $2 \mathrm{~m}$ for all photon energies. 
UNDULATOR PHASE SPACE CALCULATION

Epsx, Epsy, K2 7.27272727273E-9

Photon Wave Length: in $A$ and in $\mathrm{keV}$

Insertion Device Length 5.2

Minnimum Value of Phs at Bx By $1.34603 E-17 \quad 8.5$.

Horizontal Axis Betax: Vertical Axis Betay

Plot is normalized with Phase Space minimm

\section{$\begin{array}{lllllllllllllllllllll}0 & 1 & 2 & 3 & 4 & 5 & 6 & 7 & 8 & 9 & 10 & 11 & 12 & 13 & 14 & 15 & 16 & 17 & 18 & 19 & 20\end{array}$}

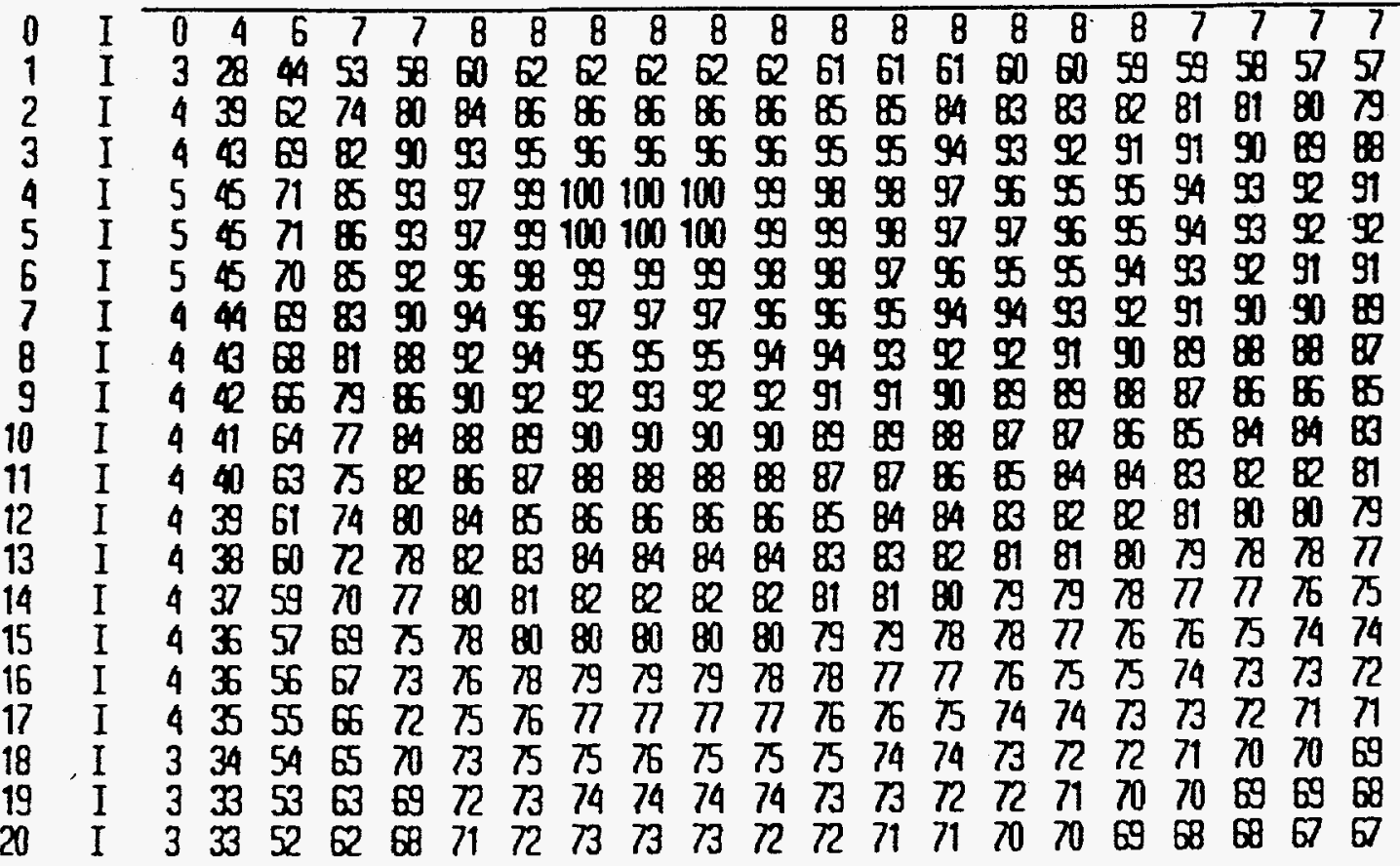

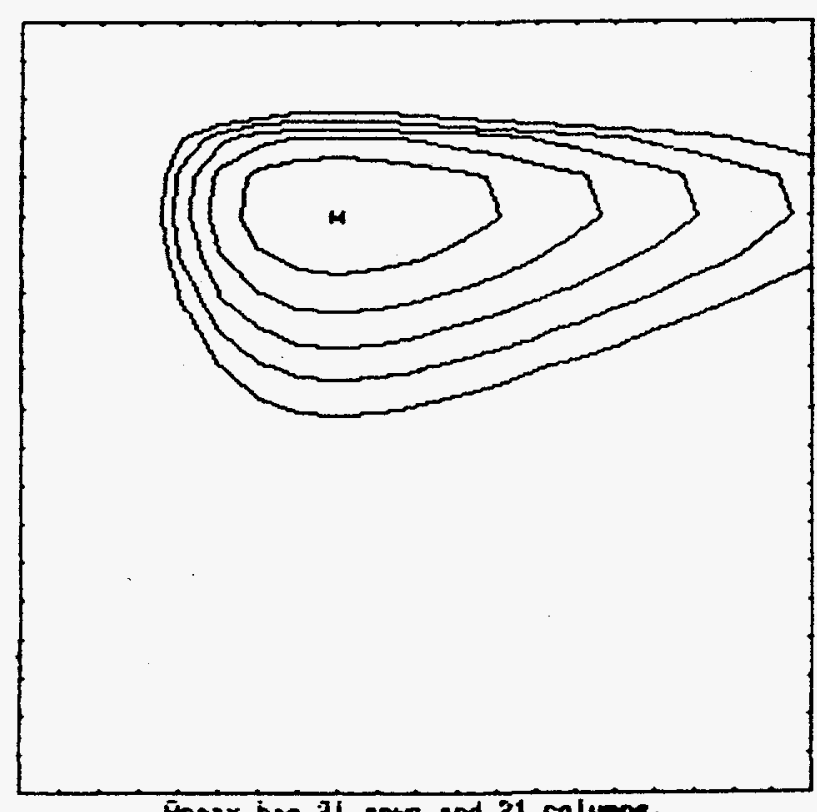

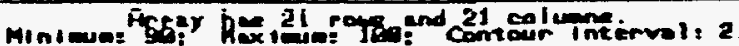
Horizontal Axis (right): Betax between 0.20 . Vertical Axis (dan) : Betay between 0.20 . 
UNDULATOR PHASE SPACE CALCULATION

$\begin{array}{ccc}\text { Epsx. Epsy, K2 7.27272727273E-9 } & 7.27272727273 \mathrm{E}-10 & \mathbf{5}^{1} \\ \text { Photon Wave Length: in } A \text { and in keV } & 2.4794 & \end{array}$

Insertion Device Length 5.2

Minnimum Value of Phs at Bx By $7.45302 \mathrm{E}-18$ 13. 7.

Horizontal Axis Betax: Vertical Pxis Betay

Plot is nornalized with Phase Spare minimm

$\begin{array}{lllllllllllllllllllll}0 & 1 & 2 & 3 & 4 & 5 & 6 & 7 & 8 & 9 & 10 & 11 & 12 & 13 & 14 & 15 & 16 & 17 & 18 & 19 & 20\end{array}$

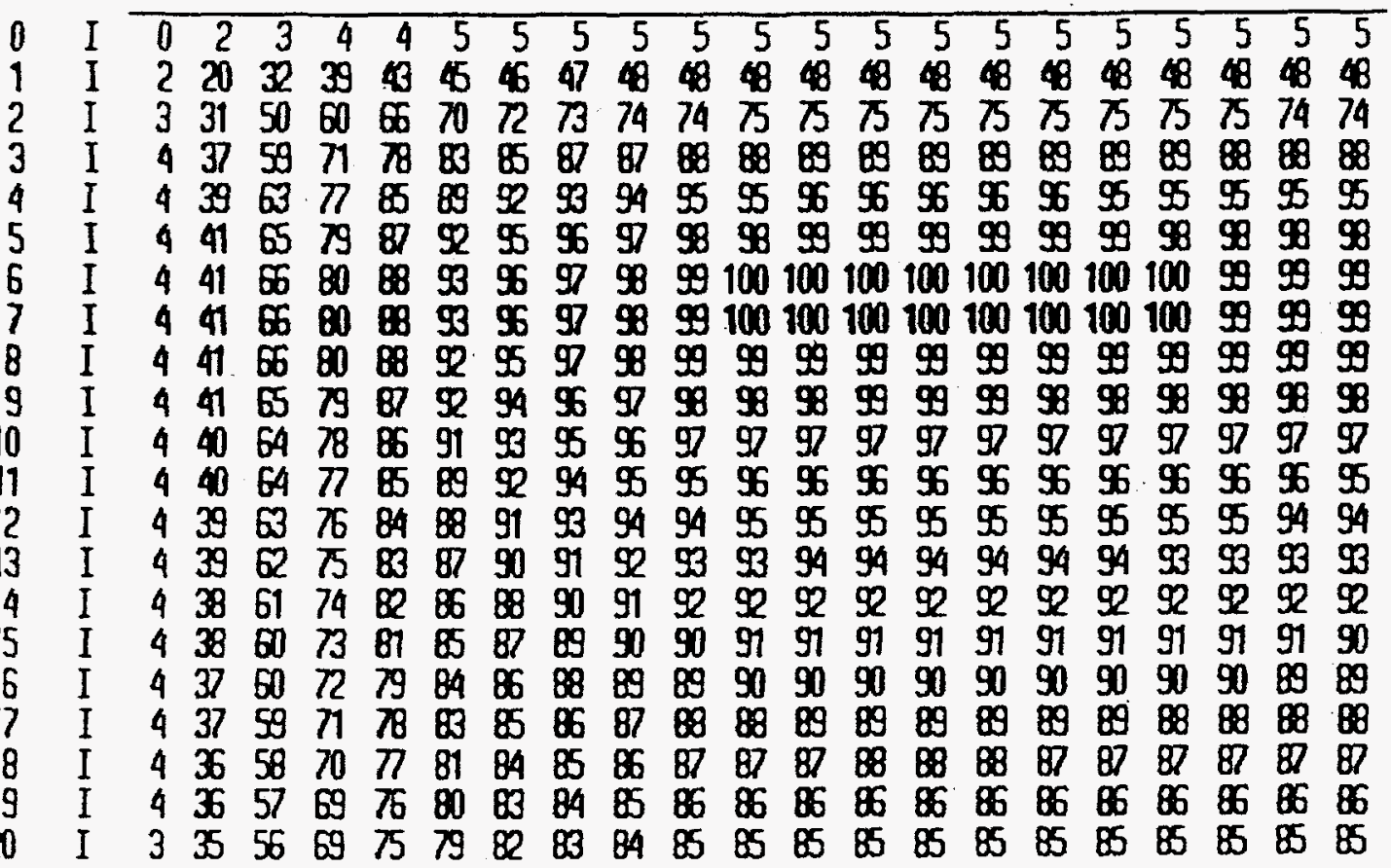

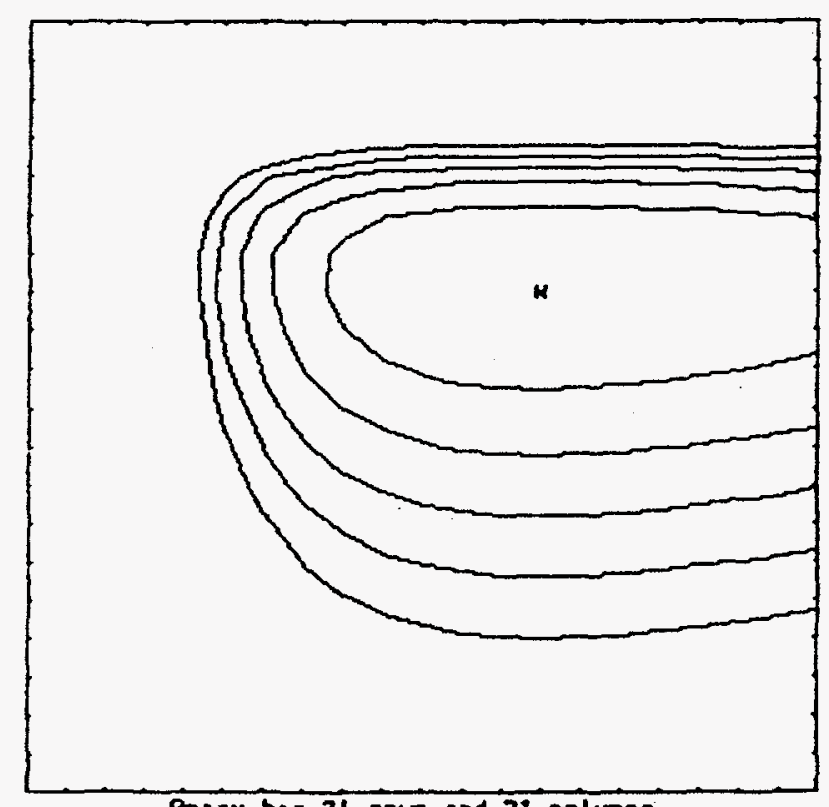

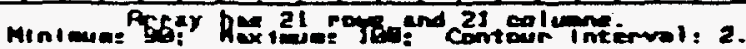

Horizontal Axis (right): Betax between 0. 20.

Vertical Axis (dom) : Betay between 0. 20. 
UNDULATOR PHASE SPACE CALCULATION

Epsx, Epsy, K2 7.27272727273E-9

Photon Wave Length: in $A$ and in $\mathrm{keV}$ $7.27272727273 \mathrm{E}-10$

1

Insertion Device Length 5.2

Minnimum Value of Phs at Bx By 6.57951E-18 16.8 .

Horizontal Pxis Betax: Vertical Axis Betay

Plot is nomalized with Ptrase Spare minimm

\section{$\begin{array}{lllllllllllllllllllll}0 & 1 & 2 & 3 & 4 & 5 & 6 & 7 & 8 & 9 & 10 & 11 & 12 & 13 & 14 & 15 & 16 & 17 & 18 & 19 & 20\end{array}$}

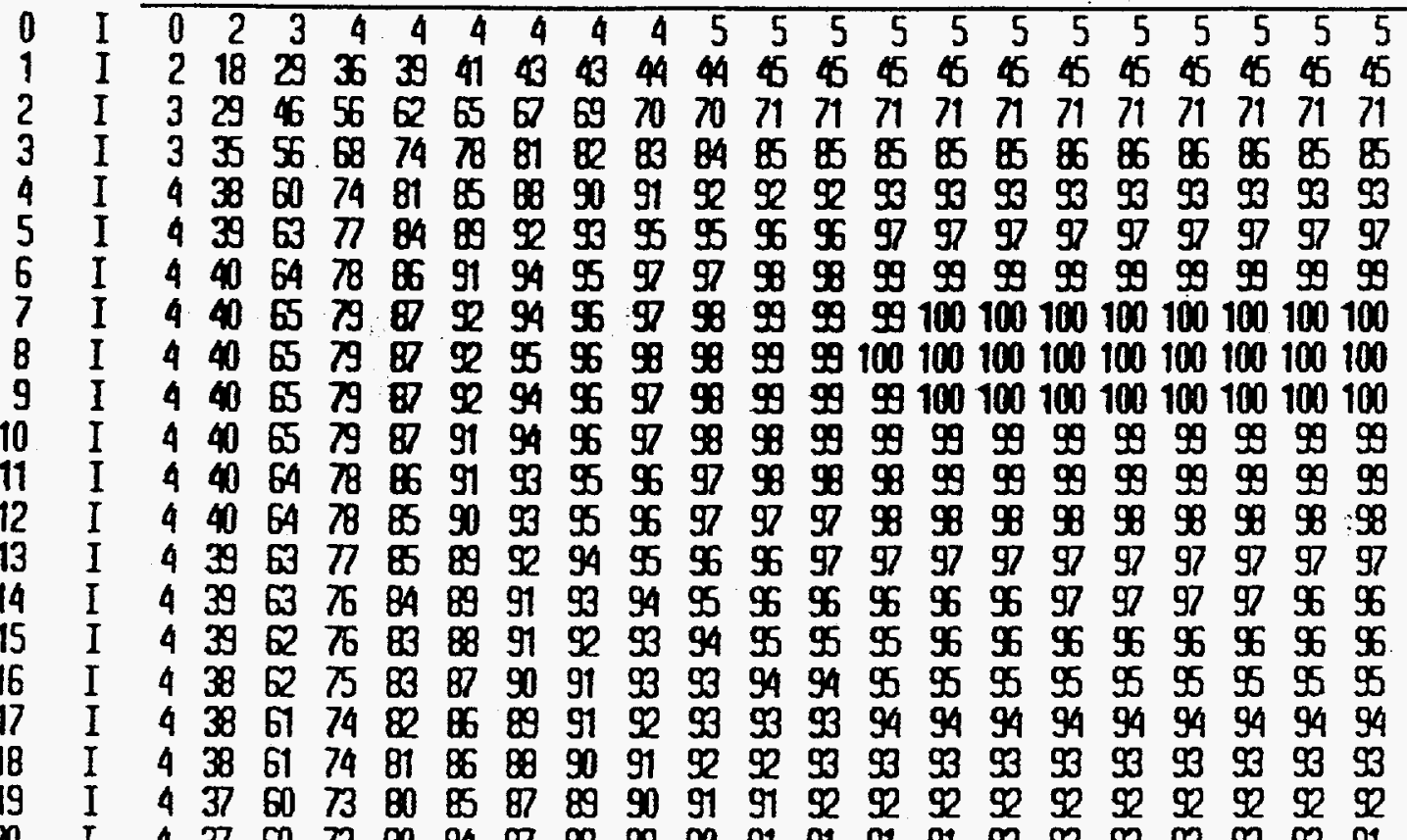

$\begin{array}{llllllllllllllllllllllll}20 & I & 4 & 37 & 60 & 72 & 80 & 84 & 8 & 88 & 89 & 90 & 91 & 91 & 91 & 91 & 92 & 92 & 92 & 92 & 92 & 92 & 91\end{array}$

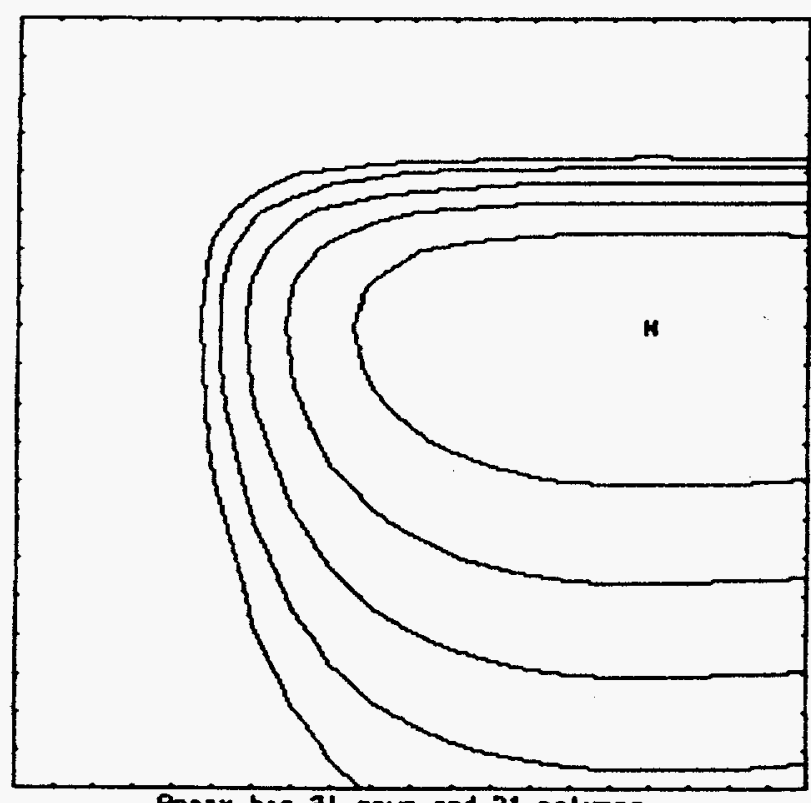

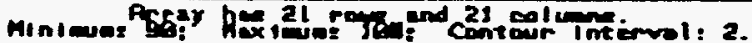
Horizontal Axis (right): Betax between 0.20 . Vertical Axis (dan) : Betay between 0. 20. 
UNDULATOR PHASE SPACE CALCULATION

$\begin{array}{ccc}\text { Epsx, Epsy, K2 } 7.27272727273 E-9 & 7.27272727273 E-10 & 1 \\ \text { Photon Wave Length: in } A \text { and in keV } & .826466666667 & \text { is }\end{array}$

Insertion Device Length 5.2

Minnimum Value of Phs at Bx By 6.25298E-18 19.9.

Horizontal Axis Betax: Vertical Axis Betay

Plot is nomalized uith Phase Space minimm

$\begin{array}{lllllllllllllllllllll}0 & 1 & 2 & 3 & 4 & 5 & 6 & 7 & 8 & 9 & 10 & 11 & 12 & 13 & 14 & 15 & 16 & 17 & 18 & 19 & 20\end{array}$

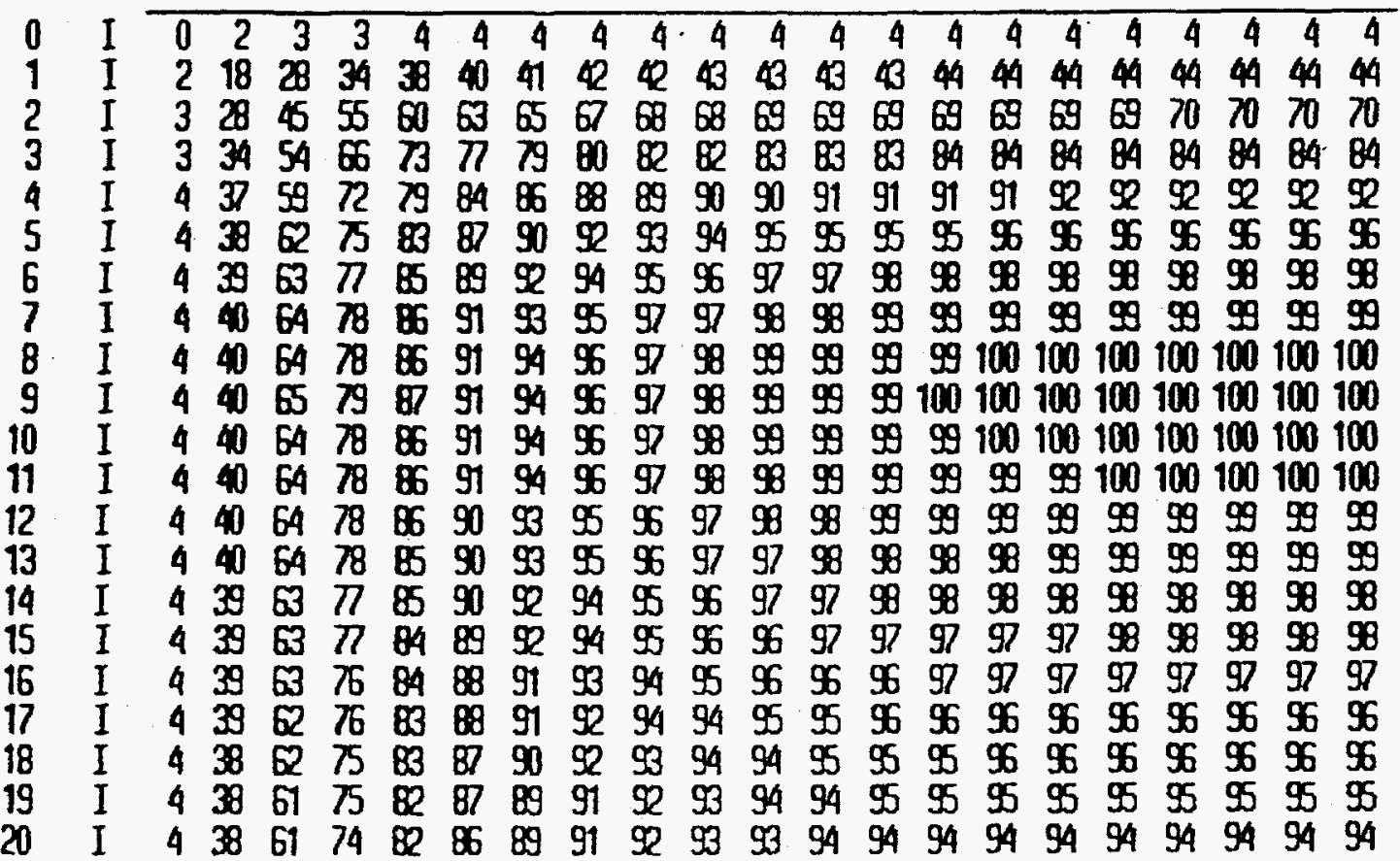

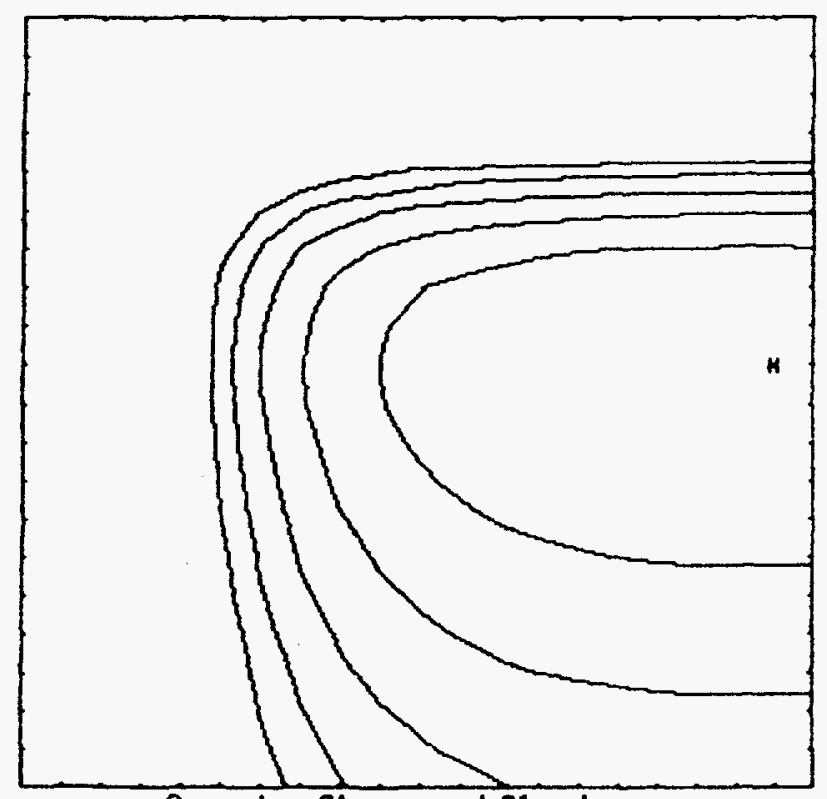

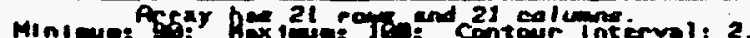

Horizontal Axis (right): Betax between 0.20 .

Vertical Axis (dan) : Betay between 0. 20.

Figure 4 
UNDULATOR PHASE SPACE CALCULATION

$\begin{array}{lcl}\text { Epsx, Epsy, K2 } 7.27272727273 E-9 & 7.27272727273 E-10 & 1 \\ \text { Photon Wave Length: in A and in kev } & .729235294118 & \text { it } \\ \text { Insertion Device Length } 5.2 & & \end{array}$

Minnimum Value of Phs at Bx By $6.17140 \mathrm{E}-18$ 19. 9.

Horizontal Axis Betax: Vertical Axis Betay

Plot is noralized with Phase Space minimm

$\begin{array}{lllllllllllllllllllll}0 & 1 & 2 & 3 & 4 & 5 & 6 & 7 & 8 & 9 & 10 & 11 & 12 & 13 & 14 & 15 & 16 & 17 & 18 & 19 & 20\end{array}$

0 I $0 \begin{array}{lllllllllllllllllllll}0 & 2 & 3 & 3 & 4 & 4 & 4 & 4 & 4 & 4 & 4 & 4 & 4 & 4 & 4 & 4 & 4 & 4 & 4 & 4 & 4\end{array}$

$\begin{array}{llllllllllllllllllllll}1 & I & 2 & 17 & 28 & 34 & 37 & 39 & 41 & 4 & 4 & 42 & 43 & 43 & 43 & 43 & 43 & 43 & 43 & 43 & 43 & 43\end{array}$

2 I 3 28 $4454 \quad 60 \quad 6365 \quad 66 \quad 67686868696969696969696969$

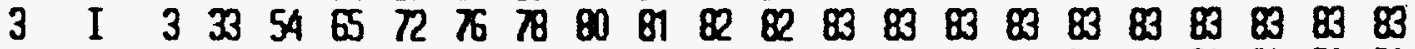

$\begin{array}{lllllllllllllllllllllll}4 & I & 4 & 36 & 59 & 72 & 79 & 83 & 86 & 87 & 89 & 89 & 90 & 90 & 91 & 91 & 91 & 91 & 91 & 91 & 91 & 91 & 91\end{array}$

$\begin{array}{llllllllllllllllllllll}5 & \text { I } 4 & 38 & 61 & 75 & 83 & 87 & 90 & 92 & 93 & 94 & 94 & 95 & 95 & 95 & 95 & 95 & 95 & 95 & 96 & 96 & 96\end{array}$

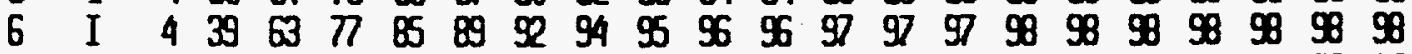

$7 \quad I \quad 4 \quad 406478$ \&6 $9093 \quad 95969898989699999999999$

8 I $4 \quad 4064 \quad 78 \quad 8691 \quad 94 \quad 9697989899999999100100100100100100$

9 I $4406478 \quad 869194969798999999100100100100100100100100$

10 I . 4 40 64 78 86 $9194 \quad 969798999999100100100100100100100$

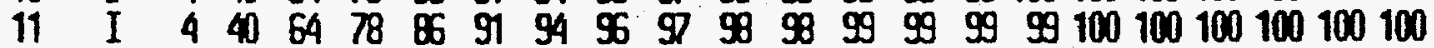

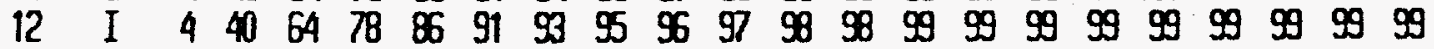

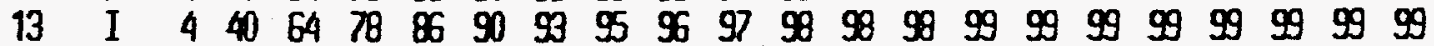

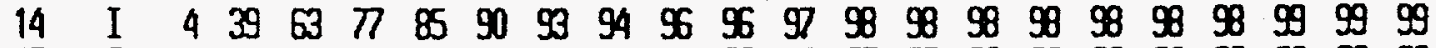

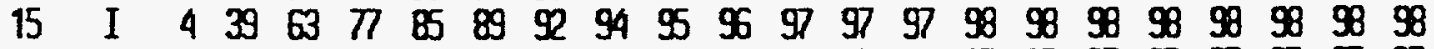

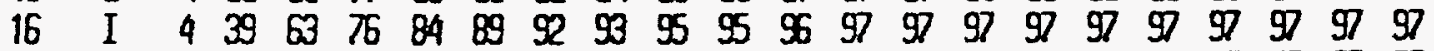

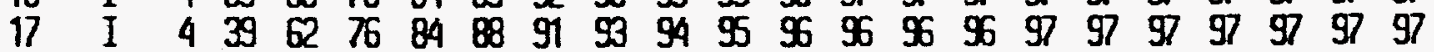

18 I $\quad \begin{array}{llllllllllllllllllllllllll}39 & 02 & 76 & 83 & 88 & 91 & 92 & 94 & 94 & 95 & 95 & 96 & 96 & 96 & 96 & 9 & 96 & 96 & 96 & 96\end{array}$

$\begin{array}{lllllllllllllllllllllllllll}19 & I & 4 & 38 & 62 & 75 & 83 & 87 & 90 & 92 & 93 & 94 & 94 & 95 & 95 & 95 & 96 & 96 & 96 & 96 & 96 & 96 & 96\end{array}$

$\begin{array}{lllllllllllllllllllllllll}20 & \text { I } & 4 & 38 & 61 & 75 & 82 & 87 & 89 & 91 & 92 & 93 & 94 & 94 & 95 & 95 & 95 & 95 & 95 & 95 & 95 & 95 & 95\end{array}$

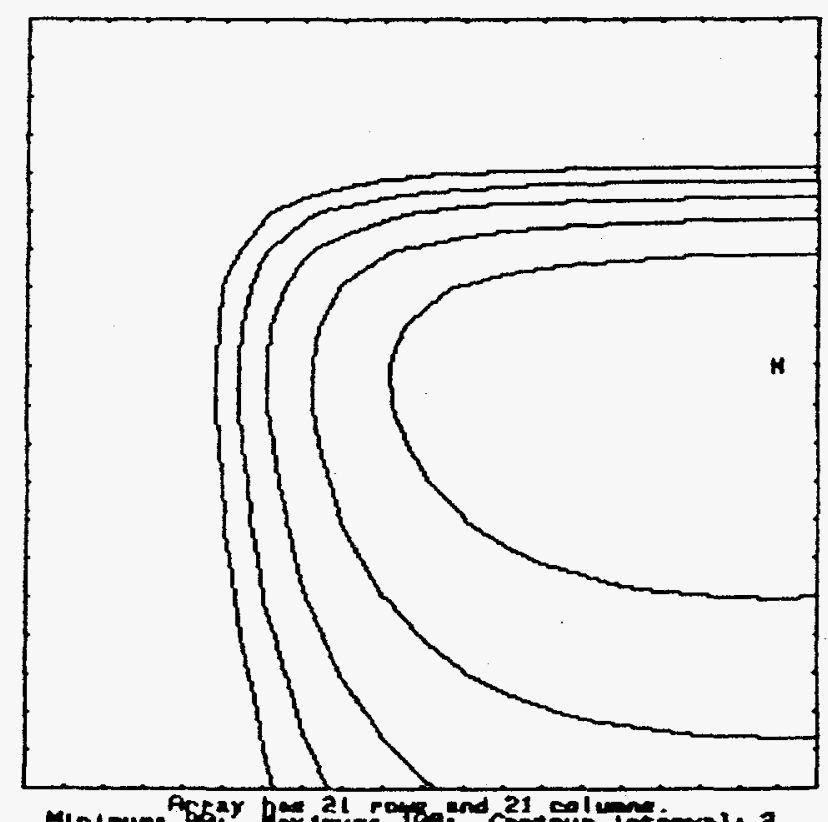

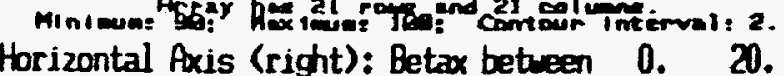

Vertical Axis (down) : Betay between $0 . \quad 20$.

Figure 5 
UNDULATOR PHASE SPACE CALCULATION

$\begin{array}{lcc}\text { Epsx, Epsy, K2 } 7.27272727273 E-9 & 7.27272727273 E-10 & 1 \\ \text { Photon Wave Length: in A and in keV } & .61985 & 20 \\ \text { Insertion Device Length } 5.2 & & \end{array}$

Minnimum Value of Phs at Bx By 6.07539E-18 20. 10.

Horizontal Axis Betax: Vertical Axis Betay

Plot is nonealized with Phase Space minimum

$\begin{array}{lllllllllllllllllllll}0 & 1 & 2 & 3 & 4 & 5 & 6 & 7 & 8 & 9 & 10 & 11 & 12 & 13 & 14 & 15 & 16 & 17 & 18 & 19 & 20\end{array}$

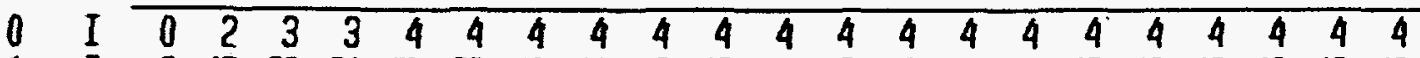

$\begin{array}{llllllllllllllllllllll}1 & 1 & 2 & 17 & 28 & 34 & 37 & 39 & 40 & 41 & 4 & Q & 4 & 42 & 43 & 43 & 43 & 43 & 43 & 43 & 43 & 43\end{array}$

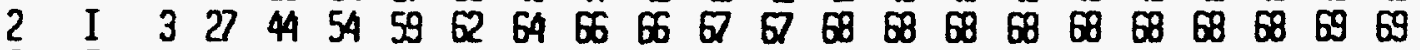

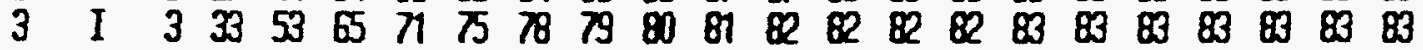

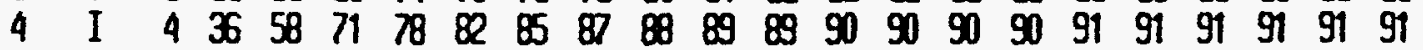

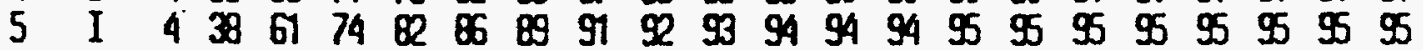

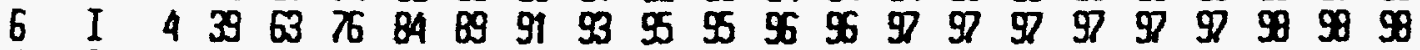

7 I 4396477 85 $90 \quad 939596 \quad 989798989999999999999999$

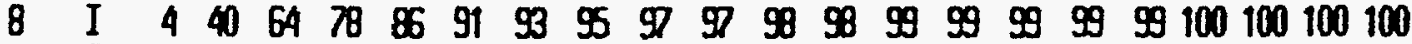

9 I 4406478 86 $9194 \quad 96979898999999100100100100100100100$

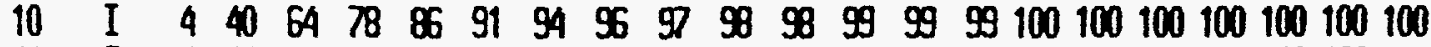

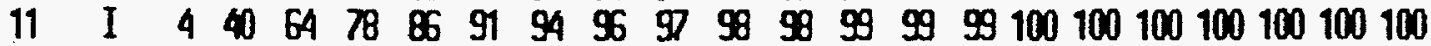

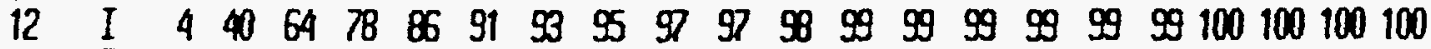

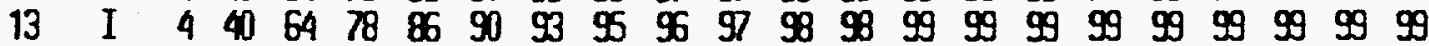

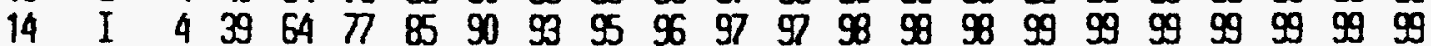

$\begin{array}{llllllllllllllllllllllllll}15 & I & 4 & 39 & 63 & 77 & 85 & 90 & 92 & 94 & 95 & 96 & 97 & 97 & 98 & 96 & 98 & 98 & 98 & 98 & 98 & 99 & 99\end{array}$

$\begin{array}{lllllllllllllllllllllllll}16 & \text { I } & 4 & 39 & 63 & 77 & 85 & 89 & 92 & 94 & 95 & 96 & 97 & 97 & 98 & 98 & 98 & 98 & 98 & 98 & 9 & 98 & 98\end{array}$

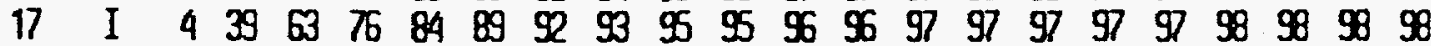

$\begin{array}{lllllllllllllllllllllll}18 & I & 4 & 39 & 62 & 76 & 84 & 88 & 91 & 93 & 94 & 95 & 96 & 96 & 96 & 97 & 97 & 97 & 97 & 97 & 97 & 97 & 97\end{array}$

$\begin{array}{lllllllllllllllllllllll}19 & I & 4 & 39 & 62 & 76 & 83 & 88 & 91 & 92 & 94 & 94 & 95 & 96 & 96 & 96 & 96 & 96 & 96 & 97 & 97 & 97 & 97\end{array}$

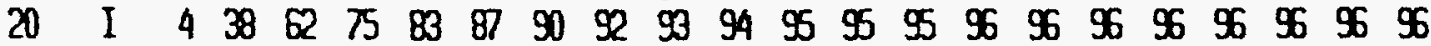

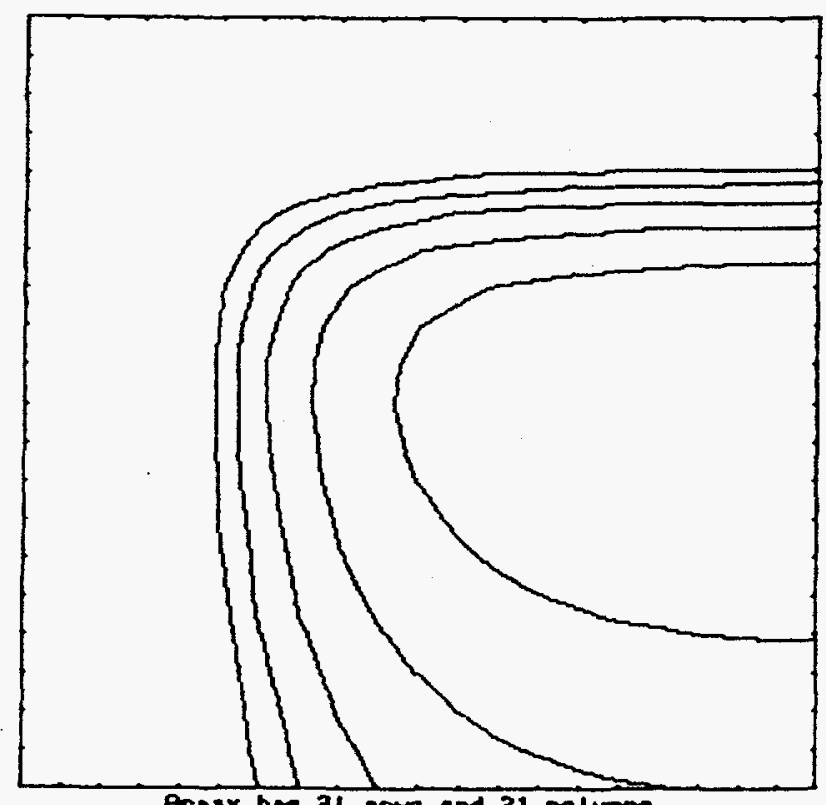

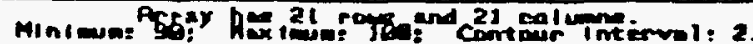

Horizontal Axis (right): Betax between 0. 20.

Vertical Axis (dan) : Betay between 0.20. 


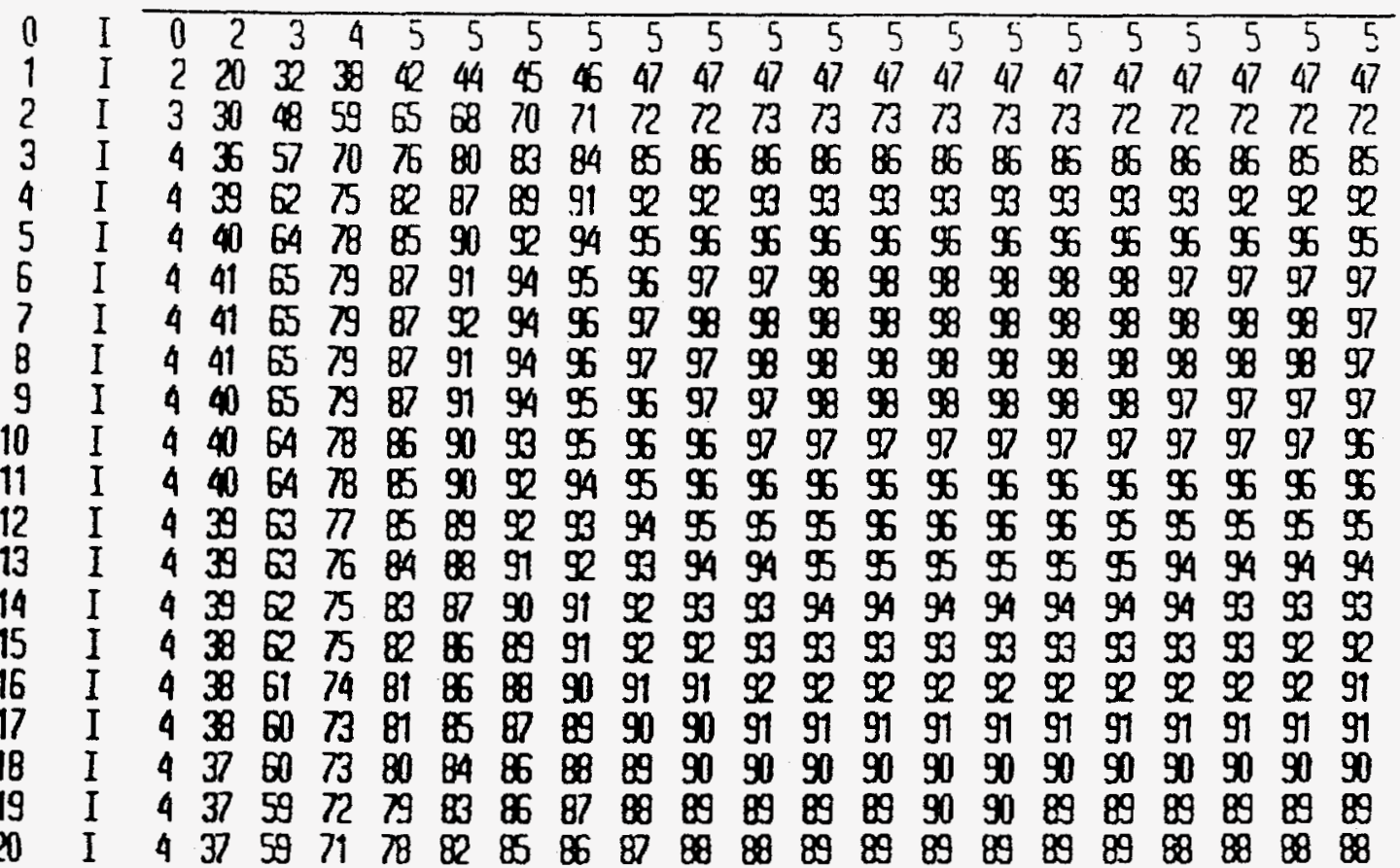

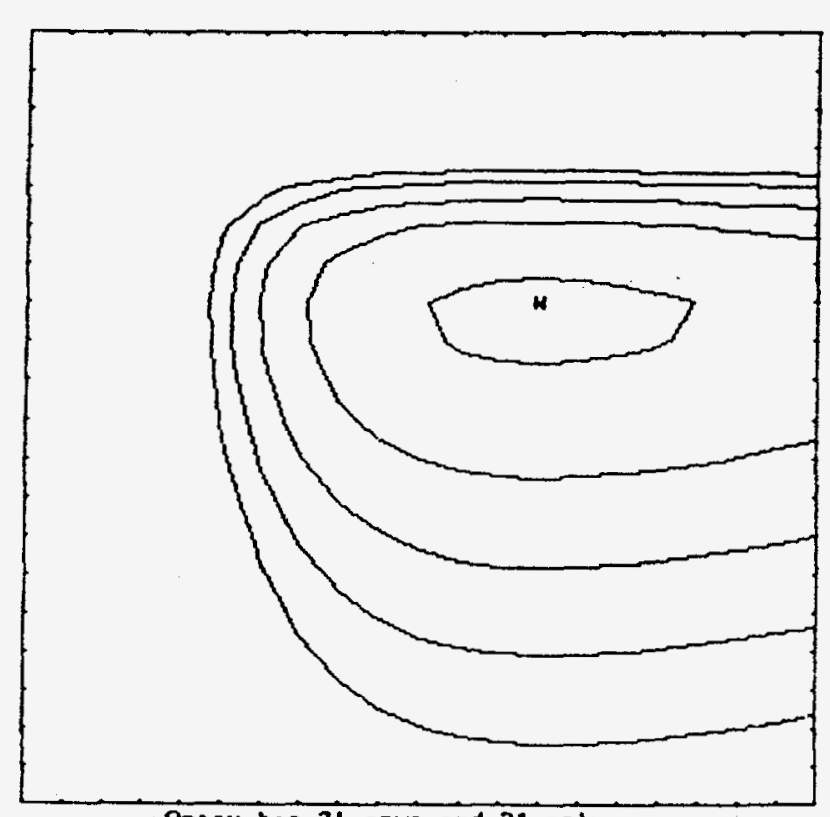

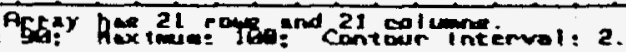

Figure 7. Sumed Contour Plot of Fig. 1-6. 
WIGGLER RADIATION PHASE SPACE CALCULATIÚN

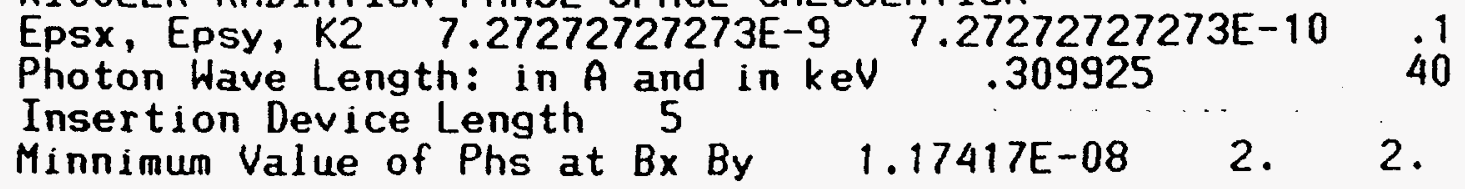

Horizontal Axis Betax: Vertical Axis Betay

Plot is nomal ized uith Phuse Space minimm

$\begin{array}{lllllllllllllllllllll}0 & 1 & 2 & 3 & 4 & 5 & 6 & 7 & 8 & 9 & 10 & 11 & 12 & 13 & 14 & 15 & 16 & 17 & 18 & 19 & 20\end{array}$

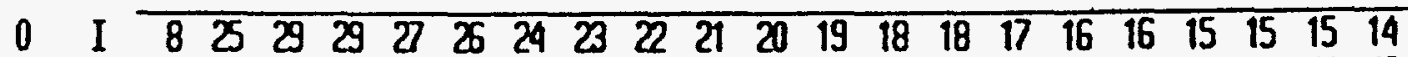

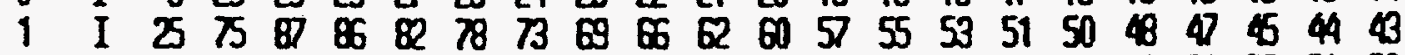

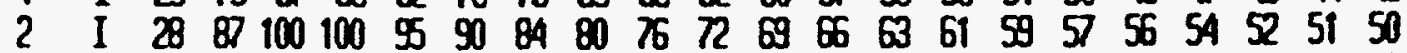

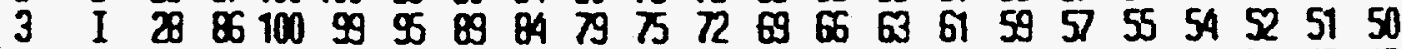

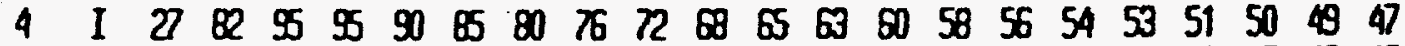

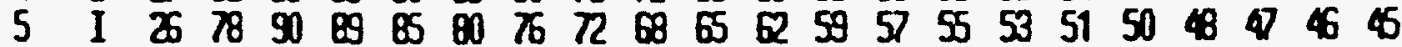

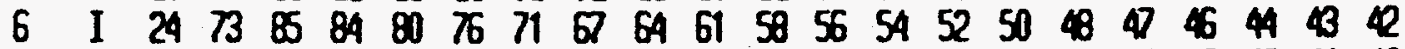

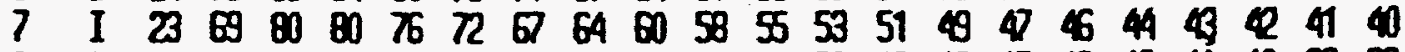

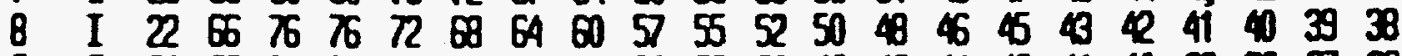

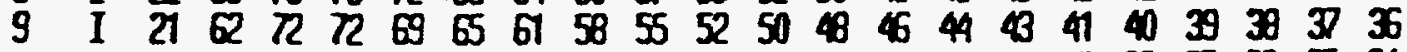

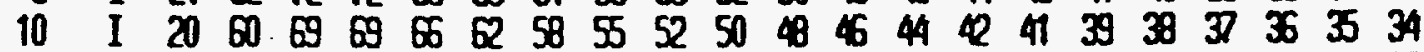

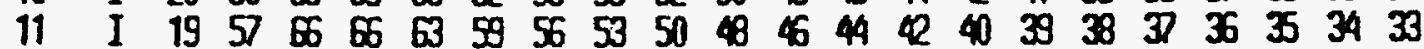

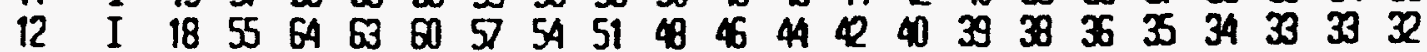

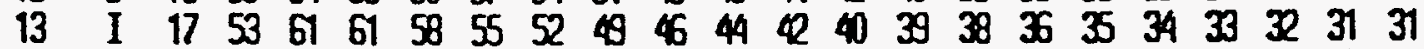

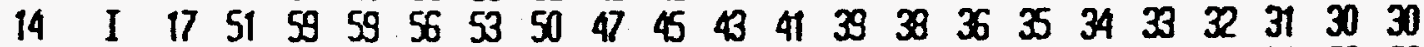

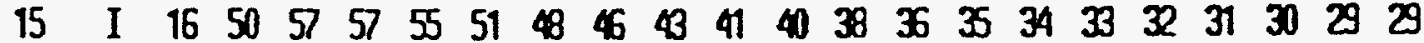

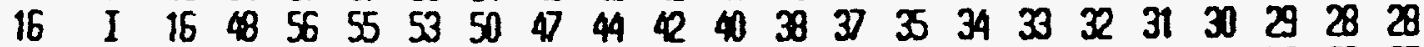

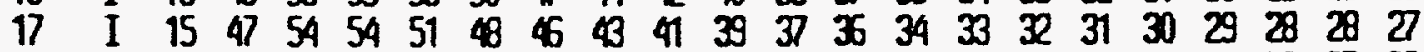

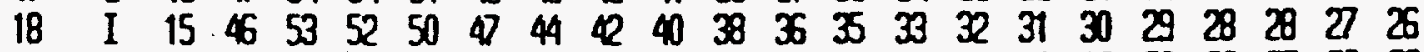

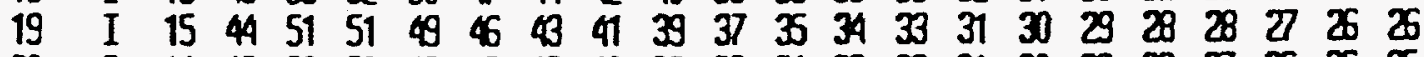

$\begin{array}{lllllllllllllllllllllllllllllllllll}20 & I & 14 & 43 & 50 & 50 & 48 & 45 & 40 & 38 & 36 & 34 & 33 & 32 & 31 & 30 & 29 & 28 & 21 & 26 & 26 & 25\end{array}$

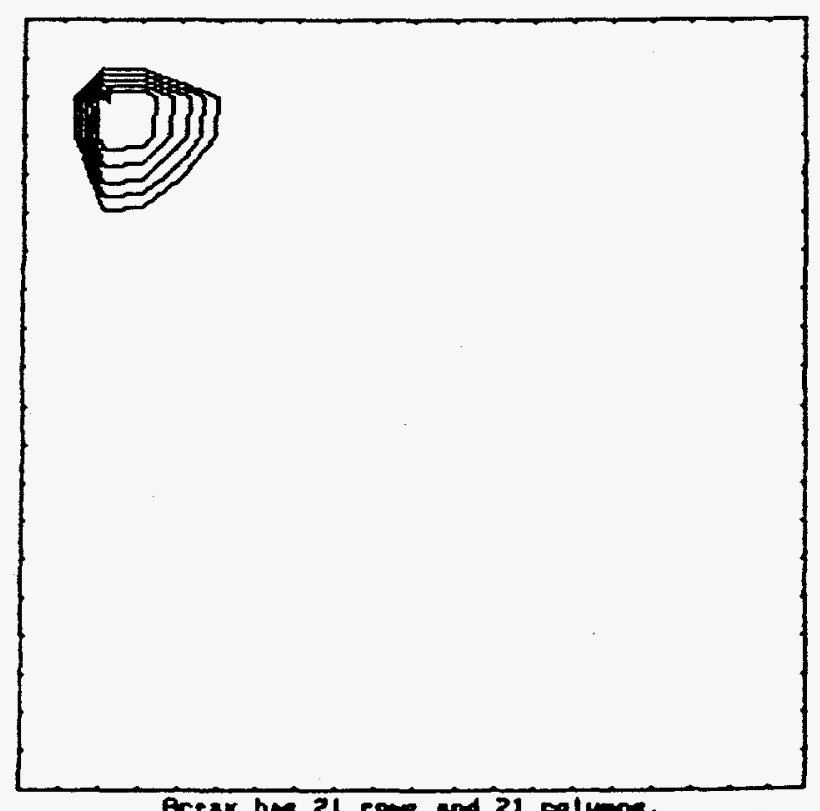

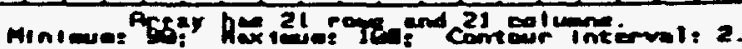
Horizontal Axis (right): Betax between 0.20 . Vertical Axis (dom) : Betay between 0.20 . 